\title{
Short implants versus bone grafting and standard-length implants placement: a systematic review
}

\author{
Juan A. V. Palacios ${ }^{1}$ - Jaime Jiménez Garcia ${ }^{2,3,4}$ • João M. M. Caramês ${ }^{3,5,6}$ • \\ Marc Quirynen $^{7}$ • Duarte Nuno da Silva Marques ${ }^{1,6,8,9}$
}

Received: 24 November 2016 / Accepted: 7 September 2017

(C) Springer-Verlag GmbH Germany 2017

\begin{abstract}
Objectives The aim of this study was to conduct a systematic review to compare the survival rates between short implants (length $<10 \mathrm{~mm}$ ) versus standard-length implants $(\geq 10 \mathrm{~mm}$ ) inserted in grafted bone. As secondary outcomes, marginal bone loss and survival rates of the implant supported prostheses were also analysed.

Materials and methods Randomised controlled trials (RCT) that compared both techniques were searched on three electronic databases till June 2016, a manual search was performed on the bibliography of the collected articles, and the authors were contacted for additional references. The estimates of the interventions were expressed in relative risk
\end{abstract}

Duarte Nuno da Silva Marques

duartemd@yahoo.co.uk

1 Máster Universitario en Implantología Avanzada Oral Avanzada, Universidad Europea de Madrid, Madrid, Spain

2 Implant Department, Universidad Europea de Madrid, Madrid, Spain

3 Periodontology and Implant Dentistry Department, New York University College of Dentistry, New York, NY, USA

4 CIRO, Madrid, Spain

5 Oral Surgery and Implant Department, LIBPhys-FCT UID/ FIS/ 04559/201, Faculdade de Medicina Dentária, Universidade de Lisboa, Lisboa, Portugal

6 Implantology Institute, Lisbon, Portugal

7 Periodontology and Oral Microbiology Department, Faculty of Medicine, Catholic University Leuven, Leuven, Belgium

8 Centro de Estudos de Medicina Dentária Baseada na Evidência, Faculdade de Medicina Dentária, Universidade de Lisboa, Lisboa, Portugal

9 LIBPhys-FCT UID/ FIS/04559/201, Faculdade de Medicina Dentária, Universidade de Lisboa, Lisboa, Portugal
(RR), mean implant survival rates and mean differences in marginal bone.

Results Eight RCTs were included in this study. From a total of 458 short implants, 15 failed (mean survival rates $=96.7 \%$ ), While from 488 regular implants, 13 failed (mean survival rates $=97.3 \%)$. The technique did not significantly affect: the implant failure rate $(P>0.05)$, with RR of $1.34(95 \% \mathrm{CI}$ $0.67-2.87)$, the mean differences of marginal bone loss $(P=0.18 ; \mathrm{MD}-0.04 \mathrm{~mm}[-0.10 ; 0.02] 95 \% \mathrm{CI})$, at loading or prosthesis failures rates (RR:0.98; 95\% CI 0.40-2.41). The mean differences of marginal bone at 1 year follow-up (post loading) presented significant marginal changes in the short implant group $(P=0.002$; $\mathrm{MD}-0.10 \mathrm{~mm}[-0.16 ;-0.03]$ $95 \% \mathrm{CI}$ ) although a significant high heterogeneity was found between groups.

Conclusions This systematic review suggests no difference between both techniques in the treatment of atrophic arches. However, more long-term RCTs are needed to evaluate the predictability at the long run.

Clinical relevance The use of short implants might be considered an alternative treatment, since it usually requires fewer surgical phases and tends to be a more affordable option.

Keywords Systematic review · Implantology · Implant length $\cdot$ Bone graft(s) $\cdot$ Bone regeneration

\section{Introduction}

Edentulism is a very prevalent condition throughout different populations $[1,2]$. Over the years, total edentulism has been dwindling thanks to a better knowledge of tooth-loss prevention techniques by both dental practitioners and the general public, falling a near 15\% since the 1940s [3]. Although total edentulism is not as prevalent as it used to be, there are still a 
large number of partially edentulous patients who are in need of tooth replacement in order to retain their oral functionality and aesthetics. This has been observed by Madhankumar et al. [4], who concluded in their epidemiological study that many patients with edentulous ridges with adjacent teeth cannot support a prostheses (range from 47.0 to $56.6 \%$ ).

In 1982, Brånemark described for the first time the use of dental implants for the rehabilitation of edentulous patients, with prosthetic survival rates at 15 years of $81 \%$ for the maxilla and $100 \%$ for the mandible [5]. Since then, implants are often used as a treatment option for total or partial edentulous patients [6] being that their success is directly related to the osteointegration process [7].

An effective way to increase the amount of bone-to-implant contact area is enlarging the implant length/diameter [8]. However, this is limited by the anatomic structures in the remnant bone, such as the inferior alveolar nerve, the lingual concavity, the maxillary sinus and the nasal cavity. For that reason, different ridge preservation and bone-regenerative techniques have been developed in order to increase the bony space in which an implant could be inserted, including sinus floor elevation and coronal bone regeneration. It has been proven that these techniques are predictable [9-11]. However, these techniques imply a higher economic cost for both the patient and the dentist, need a high degree of surgical expertise and require more surgical time and appointments. Furthermore, they are not free from complications, including infection and sinus membrane perforation in the case of the sinus floor elevation procedures [12]; soft tissue dehiscence and need for additional surgeries in the case of the bone regeneration procedures [13].

Thus, short implants defined as implants that have a length smaller than $10 \mathrm{~mm}$ were introduced advocating to be simpler and more effective for the rehabilitation of atrophic ridges [14, 15]. However, this first definition of short implants has been questioned, and different new definitions have been proposed like implants shorter than 8,7 or even $6 \mathrm{~mm}[16,17]$. Having into account this plethora of definitions, the authors of this systematic review used as a reference those implants that are less than $10 \mathrm{~mm}$ length.

Short implants can have, however, certain limitations, such as an unfavourable crown-to-implant ratio, poor aesthetics in the anterior atrophic maxilla, a difficult plaque control and the relative risk to lose faster an implant, should marginal bone loss occur, since there is no reserve in bone to implant contact.

However, consensual evidence is lacking regarding the survival rate of short implants (less than $10 \mathrm{~mm}$ ) compared to standard implants placed in grafted areas.

The aim of this systematic review was to assess whether short implants are a more predictable solution than standardlength implants when placed in a bony envelope enlarged with a grafting technique, as shown by their survival rates, marginal bone loss and prosthetic failure.

\section{Material and method}

Registration This systematic review has been registered in PROSPERO on $13 / 04 / 2015$, with the registration number CRD42015019393.

This review intends to answer the following focused question: Do short implants have better survival rates when compared with longer implants placed after bone regeneration in healthy, edentulous patients? This systematic review uses the PICO (Patient/Population, Intervention, Comparison and Outcome) question format in order to define the aim as well as the inclusion criteria. For this purpose, the analysed Population involves partially or fully edentulous patients, not affected by systemic conditions that could alter the outcomes. The determined Intervention is the placement of short implants in maxilla, mandible or both. The chosen comparison is dental implants placed in areas grafted by sinus floor elevation or vertical bone regeneration. Finally, the primary outcome was the survival rate of the implants (implants lost during study follow-up), and the incidence of prosthetic complications and mean differences of marginal bone loss defined as secondary outcomes.

Inclusion criteria In order to increase the quality of the available literature, the following inclusion criteria have been chosen: randomised controlled trials (RCT) in English, French, Portuguese or Spanish languages, made on healthy edentulous patients with at least 4 months post-loading follow-up and papers that consider short implants as those which length is less than $10 \mathrm{~mm}$ and are compared to standard implants placed in grafted areas in the same study.

Exclusion criterion Papers without an abstract or data repeated in other included studies.

A search in following databases was performed: PubMed, Lilacs and The Cochrane Library.

Terms of the search An electronic search was conducted in PubMed on 03/11/2015 with the following terms: ((("short implant"[Title/Abstract] OR "short implants"[Title/Abstract] OR "shorter implants"[Title/Abstract] OR "extra short implants"[Title/Abstract] OR "short dental implants"[Title/ Abstract])). This search was repeated on a further date (06/ 28/2016), and a new article by Thoma et al. was obtained through this method, as well as an update from an existing RCT. A further manual hand search was also conducted on the following journals: The International Journal of Periodontics and Restorative Dentistry (IJPRD), Clinical Oral Implant Research (COIR), The International Journal of Oral \& Maxillofacial Implants (IJOMI) and Journal of Oral and Maxillofacial Surgery (JOMS), but gave no new results.

The filters applied right after the initial electronic search were article types Randomized Controlled Trial; Text 
Availability: Abstract; Publication Dates: No limits have been established; Species: Humans; Languages: English, French, Portuguese, Spanish.

Data extraction Two independent reviewers used the inclusion and exclusion criteria in order to evaluate the articles obtained in the search. Any discrepancies were resolved by mutual accordance. All the corresponding authors of the identified studies were contacted in order to ascertain if additional articles or unpublished data was available. Also, the bibliographies of the selected studies were checked for additional data.

The quality of the included articles was evaluated using the Critical Appraisal Skills Programme (CASP) criteria for RCT, thus being analysed at both study and outcome level. The studies to be included needed to answer the questions 1 and 2 satisfactorily and have at least an overall punctuation of $50 \%$. The risk of bias of the included studies was analysed with the Cochrane collaboration criteria.

Data analysis All data collection was done using an Excel document that was designed to express all the data regarding the variables included in this study. Implant failure and Prosthesis failure were evaluated as a dichotomous outcome and the statistical unit was the implant. The $\mathrm{I}^{2}$ statistic was used to measure the proportion of statistical heterogeneity of the proposed outcome, and Cochran's test was used to determine the possible significance. Since heterogeneity was present, a random-effects model was selected [18]. The results were presented as relative risks (RR) with a $95 \%$ confidence interval $(\mathrm{CI})$. The data were analysed by using the statistical software OpenMeta[Analyst], for Mac, version 10.10 [http:// www.cebm.brown.edu/openmeta/]. For the continuous variable marginal bone loss, the outcome was evaluated as mean difference (MD) in milimeters with $95 \%$ Confidence intervals $(\mathrm{CI})$ and the statistical unit was the patient.

\section{Results}

Included studies The electronic search resulted in 484 papers, which were reduced to 6 papers [19-24] according to previously described methods and as presented in the PRISMA Diagram flowchart (Fig. 1). Two additional papers [25, 26] were included after repeating the search using a more recent date $(06 / 28 / 2016)$. The corresponding authors of the selected studies were contacted via email with a $90 \%$ return rate, but no additional scientific evidence could be added to this systematic review through this means.

The 484 initial papers that were obtained by using described search terms were reduced to 29 papers after the initial filtering. These papers were analysed through reading their title and abstract, which discarded 19 papers, eight of which were excluded because their follow-up was shorter than an updated version of the same study; while the other 11 papers were excluded for not complying with the established inclusion criteria. Ten papers were thoroughly read by the two reviewers, after which two of those papers were discarded for not comparing short implants $(<10 \mathrm{~mm})$ versus bone grafting techniques (Fig. 1).

The included articles were evaluated using the CASP Criteria for RCTs, thus being analysed at both study and outcome level (http://www.casp-uk.net/\#!checklists/cb36), as previously described in the Methods section and all the studies were of high quality (Table 1).

The Cochrane criteria indicated low risk of bias regarding allocation and randomization, although most of the included studies presented a performance bias since they were unable to guarantee that the patient and the practitioner were blinded (Fig. 2). This, however, can be justified by the fact that the surgical techniques compared between groups were radically different. The results, however, were in all cases analysed by a blinded examiner. Moreover, four studies were found to have a higher number of female patients in one of the groups, and three studies had a larger proportion of smokers in the control group $[19,23,25]$ while another study had more smokers in the test group [21].

A total of eight papers, published between 2009 and 2015, were included and analysed, as shown in Table 2. In three studies [19, 20, 22], both the short implant group and the regenerated bone group were divided in maxillary and mandibular subcategories. The eight trials as a whole reported on a total of 400 patients ranging from 20 to 83 years old, who received a total of 946 implants that were subjected to a follow-up ranging from 12 to 60 months in both private centres and university hospitals.

Survival rates All eight randomised controlled trials reported on the number of implant failures, from which an overall survival rate of $96.72 \%$ for short implants and $97.34 \%$ for standard-length implants was obtained. The studies which had 12 to 36 months have survival rates that range from 91.67 to $100 \%$ and from 96.70 to $100 \%$ for short and standard-length implants, respectively. The only study with a longer follow-up (5 years) reported a survival rate of $91.67 \%$ for short implants and $95.08 \%$ for standard-length implants [21] (Table 2).

Six studies used the same lengths for their respective implants, while five of the studies presented consistent lengths for standard-length implants. When comparing only those studies that presented consistent implant lengths, it was found that $6 \mathrm{~mm}$ implants had a survival rate that ranged from 97.0 to $100 \%$.

A forest plot concerning the main outcome was constructed using the obtained relative risk of the included studies. No statistical significant difference was found between both 
Fig. 1 PRISMA Flowchart diagram of the search strategy. The articles are sorted by identification, screening and eligibility

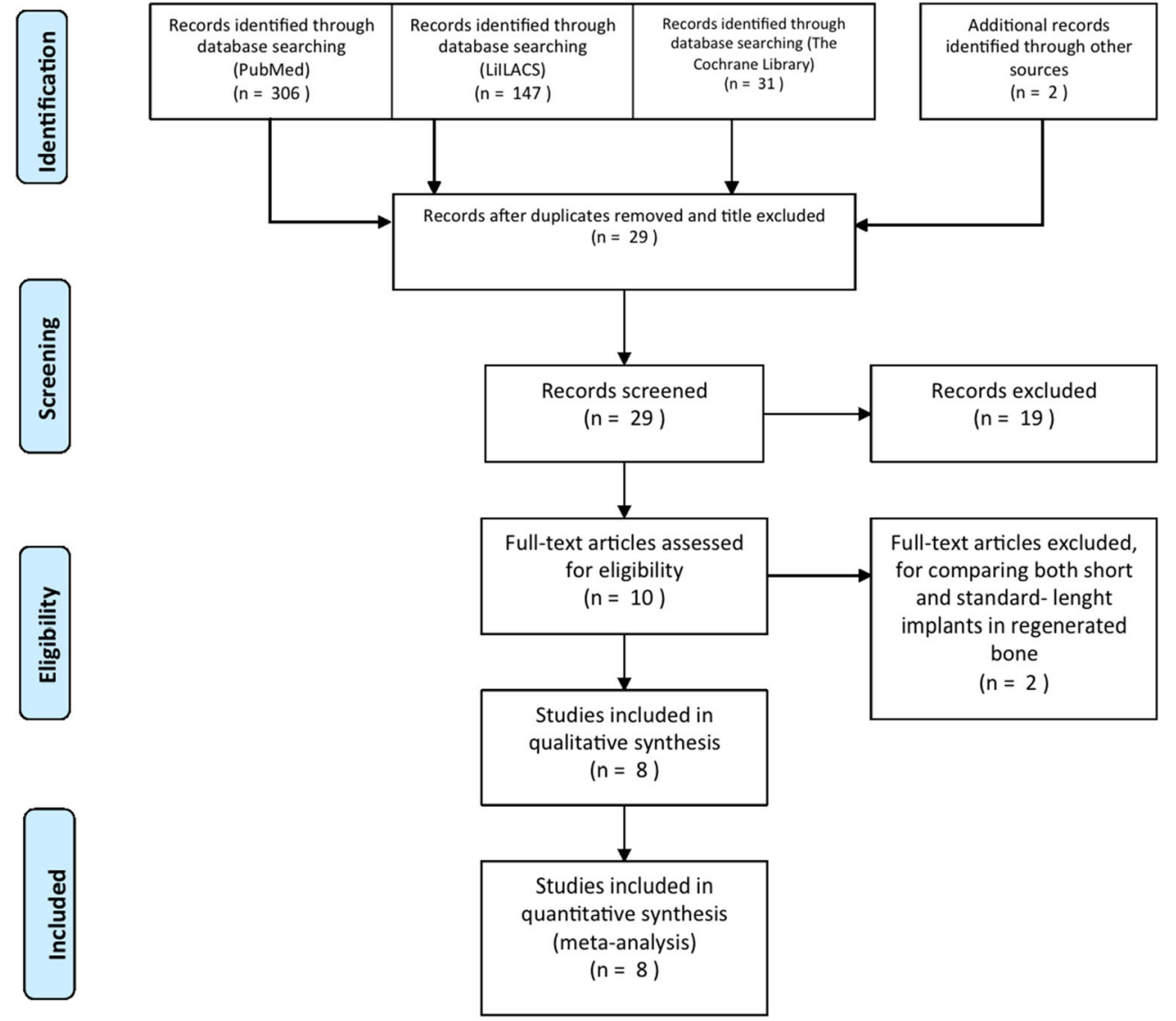

groups in overall implant survival $(P=0.45)$ with a relative risk of $1.34[0.63 ; 2.87] 95 \%$ CI, as expressed in Fig. 3a.

Additionally, the influence of the individual jaws was performed. No significant differences were obtained between the two groups in either mandible $(P=0.91 ; \mathrm{RR} 0.93[0.28 ; 3.13]$ $95 \% \mathrm{CI})$ and maxilla $(P=0.28$; RR $1.79[0.62 ; 5.16] 95 \% \mathrm{CI})$ (Fig. 3b, c).

Marginal bone loss Six studies evaluated the differences in marginal bone loss through means (mm), which were evaluated according to the study at loading, 1, 3 and 5 year followup [19-24] (Table 3). A single study [26] evaluated this outcome in different time points, at 2 weeks after loading the implant and 1 year after loading, thus not allowing the to be included in the marginal bone loss meta-analysis.

In order to be able to compare the marginal bone loss of the different groups, forest plots regarding loading and 1 year follow-up were built (Fig. 4a, b) and results indicated as mean differences of marginal bone loss and 95\% CI.

The overall analysis showed no significant differences between both groups $(P=0.18$; MD $-0.04 \mathrm{~mm}[-0.10 ; 0.02]$ $95 \% \mathrm{CI})$ for mean differences at loading and significant differences at 1 year follow-up $(P=0.002$; MD $-0.10 \mathrm{~mm}$ $[-0.16 ;-0.03] 95 \% \mathrm{CI})$. The $\mathrm{I}^{2}$ analysis of the forest plots

Table 1 Critical appraisal of the chosen articles by using the CASP tools, sorted by question number and overall number of satisfactory answers (n)

\begin{tabular}{|c|c|c|c|c|c|c|c|c|c|c|c|c|}
\hline Article & 1 & 2 & 3 & 4 & 5 & 6 & 7 & 8 & 9 & 10 & 11 & $\mathrm{n}$ \\
\hline Pistilli, Felice, Piattelli, Esposito & $\mathrm{Y}$ & $\mathrm{Y}$ & $\mathrm{N}$ & $\mathrm{N}$ & $\mathrm{Y}$ & $\mathrm{Y}$ & $\mathrm{Y}$ & $\mathrm{Y}$ & $\mathrm{Y}$ & Y & Y & 9 \\
\hline Pistilli, Felice, Cannizzaro, Esposito & $\mathrm{Y}$ & $\mathrm{Y}$ & $\mathrm{N}$ & $\mathrm{Y}$ & $\mathrm{Y}$ & $\mathrm{Y}$ & $\mathrm{Y}$ & $\mathrm{Y}$ & $\mathrm{Y}$ & Y & $\mathrm{Y}$ & 10 \\
\hline Felice, Cannizaro, Barausse, Esposito & $\mathrm{Y}$ & $\mathrm{Y}$ & $\mathrm{N}$ & $\mathrm{Y}$ & $\mathrm{Y}$ & $\mathrm{Y}$ & Y & $\mathrm{Y}$ & $\mathrm{Y}$ & Y & Y & 10 \\
\hline Esposito, Pistilli, Felice & $\mathrm{Y}$ & $\mathrm{Y}$ & $\mathrm{N}$ & $\mathrm{N}$ & $\mathrm{Y}$ & $\mathrm{Y}$ & $\mathrm{Y}$ & $\mathrm{Y}$ & $\mathrm{Y}$ & Y & $\mathrm{Y}$ & 9 \\
\hline Guljé, Meijer & $\mathrm{Y}$ & $\mathrm{Y}$ & $\mathrm{N}$ & $\mathrm{Y}$ & $\mathrm{Y}$ & $\mathrm{Y}$ & $\mathrm{Y}$ & $\mathrm{Y}$ & $\mathrm{Y}$ & Y & $\mathrm{Y}$ & 10 \\
\hline Felice, Pistilli, Esposito & $\mathrm{Y}$ & $\mathrm{Y}$ & $\mathrm{N}$ & $\mathrm{Y}$ & $\mathrm{Y}$ & $\mathrm{Y}$ & $\mathrm{Y}$ & $\mathrm{Y}$ & $\mathrm{Y}$ & Y & $\mathrm{Y}$ & 10 \\
\hline Esposito, Barausse, Pistilli & $\mathrm{Y}$ & $\mathrm{Y}$ & $\mathrm{N}$ & $\mathrm{Y}$ & $\mathrm{Y}$ & $\mathrm{Y}$ & $\mathrm{Y}$ & $\mathrm{Y}$ & $\mathrm{Y}$ & Y & $\mathrm{Y}$ & 10 \\
\hline Thoma, Hämmerle & $\mathrm{Y}$ & $\mathrm{Y}$ & $\mathrm{N}$ & $\mathrm{Y}$ & $\mathrm{Y}$ & $\mathrm{Y}$ & $\mathrm{Y}$ & $\mathrm{Y}$ & $\mathrm{Y}$ & $\mathrm{Y}$ & $\mathrm{Y}$ & 10 \\
\hline
\end{tabular}




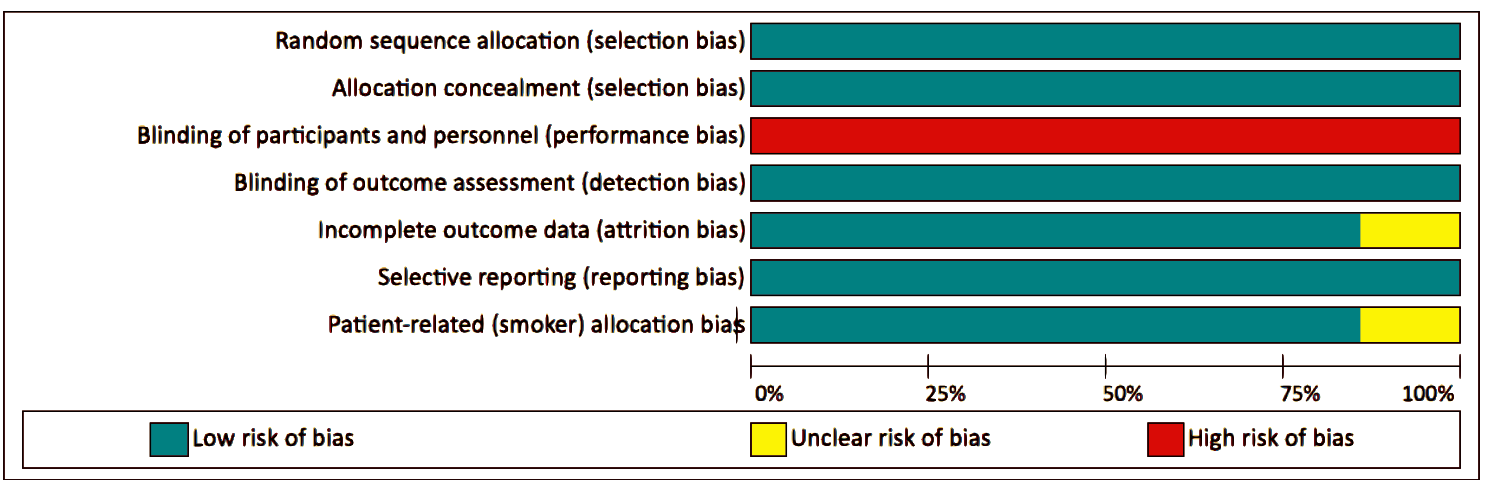

Fig. 2 Risk of bias assessment by Cochrane risk of bias tools

indicated high heterogeneity between studies with values equating between 87 and 92\% (Fig. 4a, b). Although, two of the included studies $[19,20]$ presented the results of mean differences of marginal bone loss discriminated by arch an overall MBL was not presented so both jaws were integrated in the forest plot analysis.

When performing sub-group analysis for the individual jaw, significant differences in marginal bone loss were observed at loading $(P=<0.01 ; \mathrm{MD}-0.11 \mathrm{~mm}[-0.13 ; 0.09]$ 95\% CI) as well as at 1-year follow-up $(P<0.01$; MD $-0.15 \mathrm{~mm}[-0.28 ; 0.01] 95 \% \mathrm{CI})$ for the maxilla. Although these results should be interpreted with caution due to the small number of studies (4 and 5, respectively), sample size and high heterogeneity.

Only four studies reported the mean differences between baseline-loading and baseline-1 year after loading for the mandible [19-22]. No significant differences were observed at loading $(P=0.21 ; \mathrm{MD} 0.03[-0.02 ; 0.07] 95 \% \mathrm{CI})$, neither at 1 year after loading $(P=0.57$ MD $-0.02[-0.11 ; 0.06]$ 95\% CI).

A meta-regression evaluating the relationship of mean differences of marginal bone loss during loading yielded statistical significance $(P<0.001)$. Thus, the greater the follow-up period after loading, the bigger the differences of MBL between groups favouring short implants (Fig. 5). However, it is important to mention that one of the greatest limitations of the present systematic review was the scarcity of long-term follow-up RCTs with only 1 study with a 3-year follow-up and 1 with a 5-year follow-up.

Prosthetic failure Prosthetic failures were evaluated by seven studies [19-24, 26]. An overall number of 9 short implantsupported and 10 standard-length implant-supported prostheses failed. The analysis considered, the number of events of prostheses failures (could not be repaired or that failed together with the implant) per number of implants placed. No significant differences were observed $(P=0.82$; RR $0.90[0.38$; 2.14] 95\% CI) between groups (Fig. 6).

\section{Discussion}

Main results With the available data, the results suggest that short implants perform clinically as well as longer implants, placed in augmented bone at least in the short term. Moreover, there were no differences between both groups about the implant survival, overall mean differences of marginal bone at loading and prosthetic failure rates. However, some significant differences were found in mean differences of marginal bone when comparing the 1 year post-loading or when comparing the maxilla, favouring short implants. This type of implants, furthermore, might prove to be a better option from a patient's comfort and economy-oriented point of view [25]. The authors of this systematic review consider that economical factors and patient satisfaction of both procedures could have a higher impact in an evidence-based, patient-oriented decision.

To our knowledge, this is the first systematic review that compares short implants with the use of standard implants placed in augmented bone that was performed exclusively with randomised controlled trials, for these studies provide the highest level of scientific evidence. Other systematic reviews have already addressed part of the subject [27], but the authors of the present review consider that it its justified to compile and to analyse RCTs regarding both sinus floor elevation and vertical regeneration of the maxilla or the mandible, as these techniques serve the same purpose of grafting a bone area that will receive one or more dental implants.

The chosen outcomes have been the survival rates of both types of implants as the main outcome and mean differences of marginal bone loss and incidence of prosthetic complications as secondary outcomes.

While there are some differences between the various published RCTs on this subject that can be considered as confounding factors, as different types of grafts, regeneration procedures, implant types, diameters and lengths of the implants used, when performing the quality and bias assessment, all the included studies presented high quality and most of them with low risk of bias. However, it has to be noted that a greater number of 


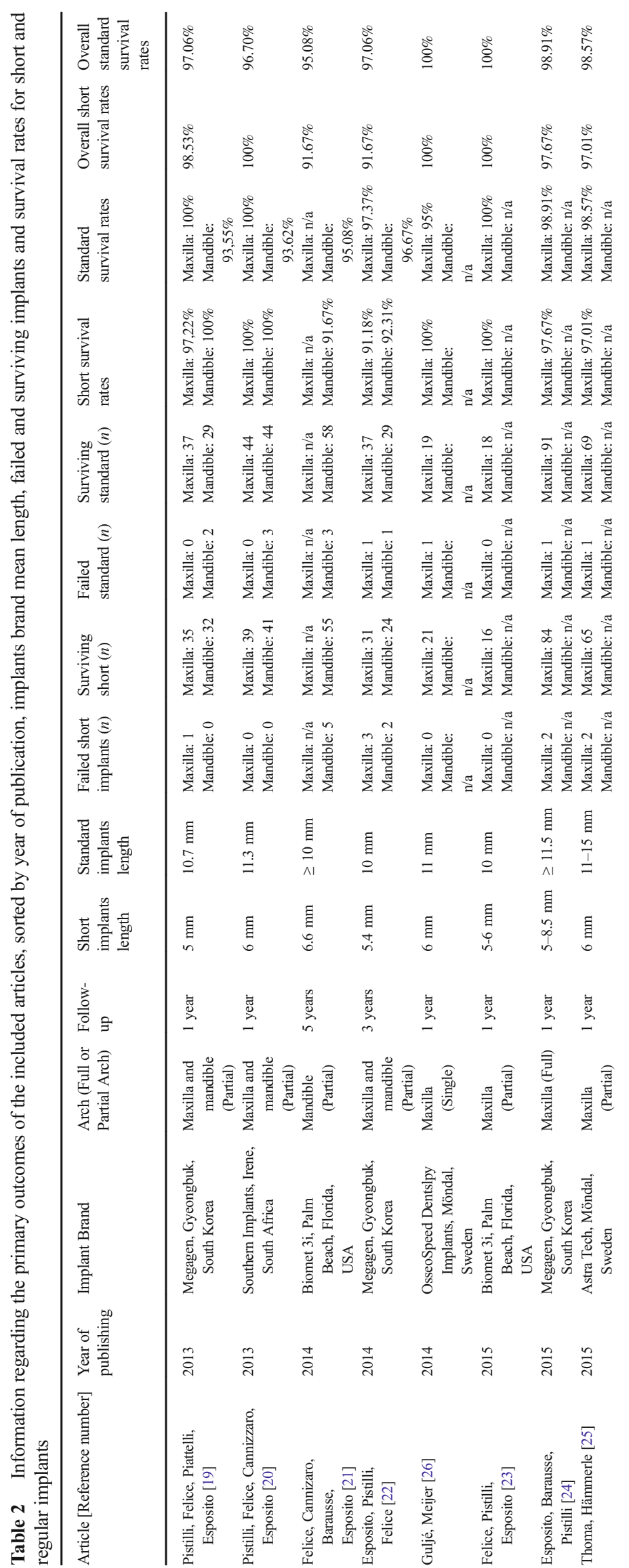




\section{a}

Studies

Pistilli, Felice, Piatelli, Esposito 2013

Pistilli, Felice, Cannizzaro, Esposito 2013

Felice, Cannizaro, Barausse, Esposito 2014

Esposito, Pistilli, Felice 2014

Gulj., Meijer 2014

Felice, Pistilli, Esposito 2015

Esposito, Barausse, Pistilli 2015

Thoma, Hammerle 2015

Overall $(95 \% \mathrm{Cl})(\mathrm{P}=0.45)$

\begin{abstract}
Estimate (95\% C.I.) Ev/Trt Ev/Ctrl
$0.500(0.046,5.385) \quad 1 / 68$

$0.162(0.009,3.094) 0 / 80$

$1.694(0.424,6.778) 5 / 60$

$2.833(0.571,14.071) 5 / 60$

$0.318(0.014,7.383) \quad 0 / 21$

$1.118(0.023,53.312) \quad 0 / 16$

$2.140(0.198,23.173) \quad 2 / 86$

$2.090(0.194,22.509) \quad 2 / 67$

$1.340(0.625,2.870) 15 / 458$

$13 / 488$
\end{abstract}

b

Studies

Estimate (95\% C.I.) EV/Trt Ev/Ctrl

Pistilli, Felice, Piatelli, Esposito 2013 Pistilli, Felice,Cannizzaro, Esposito 2013

Felice, Cannizaro, Barausse, Esposito 2014

Esposito, Pistilli, Felice 2014

$0.194(0.010,3.884) \quad 0 / 32$

$0.163(0.009,3.070) 0 / 41$

$1.694(0.424,6.778) \quad 5 / 60$

$2.308(0.222,24.012) 2 / 26$

Overall $(95 \% \mathrm{CI})(\mathrm{P}=0.91)$

$0.930(0.276,3.130) \quad 7 / 159$

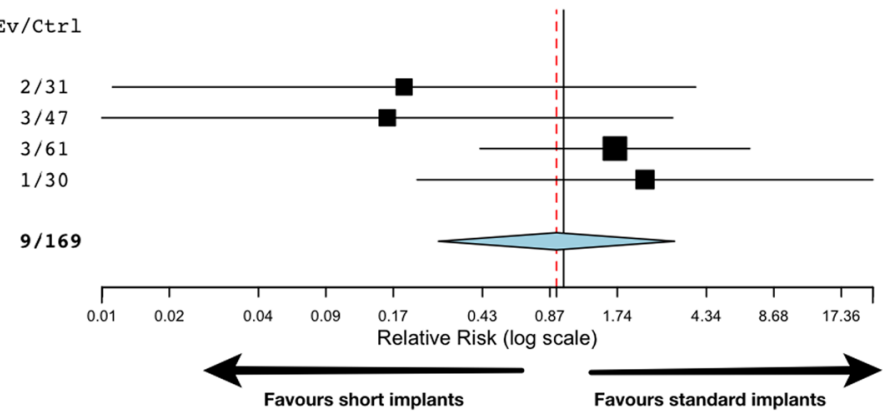

C

Studies

Pistilli, Felice, Piatelli, Esposito 2013 Pistilli, Felice, Cannizzaro, Esposito 201 Esposito, Pistilli, Felice 2014

Gulj., Meijer 2014

Felice, Pistilli, Esposito 2015

Esposito, Barausse, Pistilli 2015

Thoma, Hammerle 2015

Overall $(95 \% \mathrm{Cl})(\mathrm{P}=\mathbf{0 . 2 8})$
Estimate (95\% C.I.) Ev/Trt Ev/Ctrl

$3.081(0.130,73.238) \quad 1 / 36$

$1.125(0.023,55.396) \quad 0 / 39$

$3.353(0.366,30.729) 3 / 34$

$0.303(0.013,7.021) 0 / 21$

$1.118(0.023,53.312) \quad 0 / 16$

$2.140(0.198,23.173) \quad 2 / 86$

$2.090(0.194,22.509) \quad 2 / 67$

$1.788(0.620, \quad 5.158) \quad 8 / 299$
Fig. 3 Forest plot for survival rates of the included studies. a Overall analysis: Heterogeneity: $\mathrm{Tau}^{2}=0.00 ; \mathrm{Chi}^{2}=4.67, \mathrm{df}=7(P=0.70)$; $\mathrm{I}^{2}=0 \%$, and individualised analysis of mandible. b Heterogeneity: $\mathrm{Tau}^{2}=0.30 ; \mathrm{Chi}^{2}=3.66, \mathrm{df}=3(P=0.30) ; \mathrm{I}^{2}=17.97 \%$ and maxilla. $\mathbf{c}$ Heterogeneity: $\mathrm{Tau}^{2}=0.00 ; \mathrm{Chi}^{2}=1.80, \mathrm{df}=6(P=0.94) ; \mathrm{I}^{2}=0 \% . \mathrm{Ev} /$

female patients and smokers have been found in one of the two groups in four [19, 21, 23, 26] and three [19, 23, 25] studies, respectively, which could be considered a possible source of confounding factors. For the female patients, a recent study by Koszuta et al. [28] reported that, while gender itself does not affect the amount of implant failures, the patients receiving hormonal therapy are at a greater risk of losing their implants. As for smokers, recent studies [29, 30] have pointed that the habit of smoking has a harmful effect upon the surrounding bone, thus compromising the osseointegration.

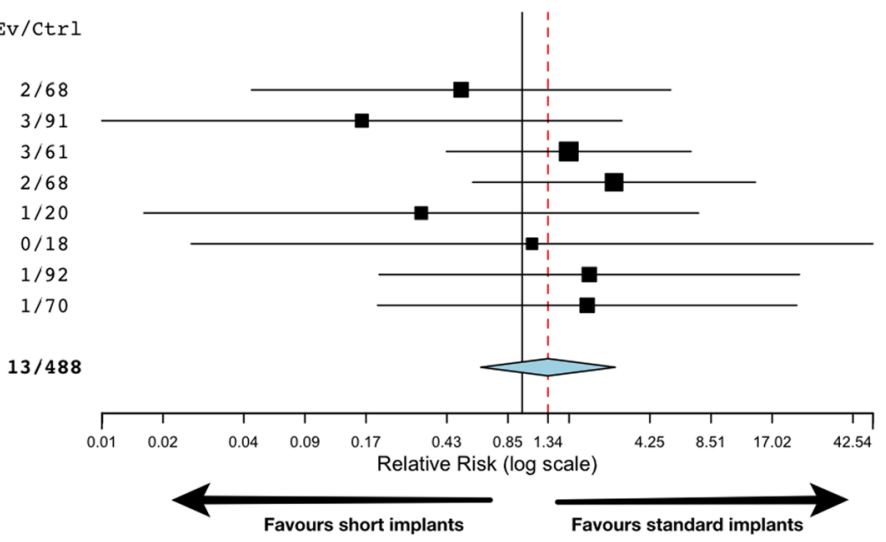

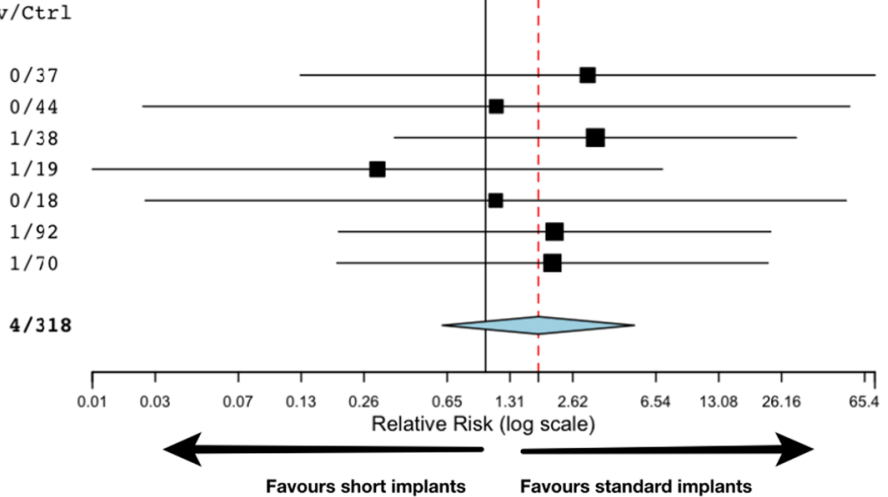

Trt represents the test (short implants) group, while Ev/Ctrl represents the control (standard implants placed in grafted bone) group. The red line represents the average of all the results, and the vertical black line represents the no-effect line

The studied population in all eight articles was comprised of standard-homogenous patients without any remarkable particularity, and the interventions were carried in both private dental offices and university hospitals, however caution is recommended when extrapolating these results to other clinical settings, since experienced clinicians performed most of the procedures evaluated in the trials.

While evaluating if sample size determination was performed in the included RCTs, it was noted that only three of the included studies had a sample size that was large enough 


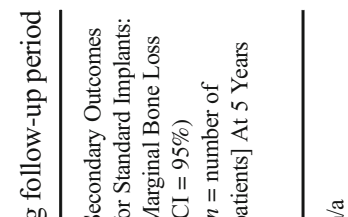

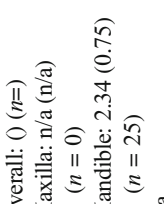

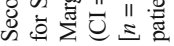

造造总

$\stackrel{\frac{1}{2}}{2}$

ठำ

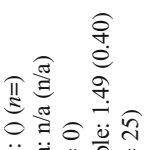

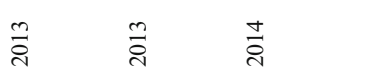

离

ำ

ำ

ำ 
a

Studies

Pistilli, Felice, Piatelli, Esposito (maxilla) 2013 Pistilli, Felice, Piatelli, Esposito (mandible) 2013 Pistilli, Felice,Cannizzaro, Esposito (maxilla) 2013 Pistilli, Felice,Cannizzaro, Esposito (mandible) 2013 Felice, Cannizaro, Barausse, Esposito 2014 Esposito, Pistilli, Felice 2014

Felice, Pistilli, Esposito 2015

Total $(95 \% \mathrm{Cl})(\mathrm{P}=\mathbf{0 . 1 8})$
Estimate ( $95 \%$ C.I.)

$-0.120(-0.152,-0.088)$

$-0.010(-0.039,0.019)$

$-0.100(-0.128,-0.072)$

$0.040 \quad(0.009,0.071)$

$0.020(-0.131,0.171)$

$-0.020(-0.100,0.060)$

$-0.050(-0.184,0.084)$

$-0.039(-0.096,0.018)$

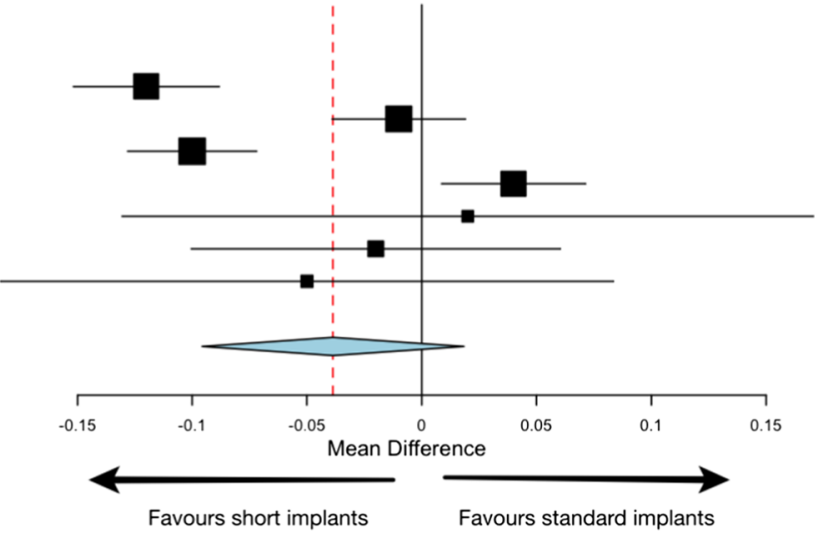

b

Studies

Estimate ( $95 \%$ C.I.)

Pistilli, Felice, Piatelli, Esposito (maxilla) 2013

Pistilli, Felice, Piatelli, Esposito (mandible) 2013

$-0.280(-0.342,-0.218)$

$-0.090(-0.129,-0.051)$

$-0.070(-0.104,-0.036)$

$-0.020(-0.059,0.019)$

$0.000(-0.172,0.172)$

$-0.190(-0.449,0.069)$

$-0.170(-0.317,-0.023)$

$0.040(-0.093,0.173)$

$-0.096(-0.163,-0.029)$

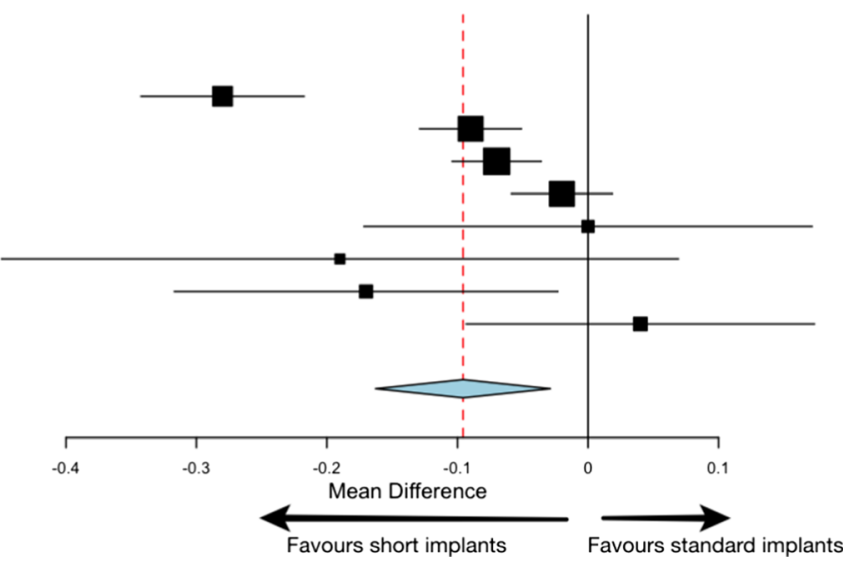

Fig. 4 Forest plot for mean differences of marginal bone changes of the included studies. a Loading: Heterogeneity: $\mathrm{Tau}^{2}=0.005 ; \mathrm{Chi}^{2}=70.90$, df $=6(P<0.001) ; \mathrm{I}^{2}=91.538 \%$, b 1 year post-loading Heterogeneity:

$\mathrm{Tau}^{2}=0.06 ; \mathrm{Chi}^{2}=55.23, \mathrm{df}=7(P<0.001) ; \mathrm{I}^{2}=87.33 \%$. The red line represents the average of all the results, and the vertical black line represents the no-effect line

When comparing the results for the primary outcome, no significant differences were found between short implants and standard implants in regenerated bone for the overall survival rates and when comparing both jaws. However, when analysing individual studies, it was possible to see that smaller length implants (bellow $7 \mathrm{~mm}$ ) present lower survival rates.

Fig. 5 Meta-regression for mean differences in marginal bone loss $(\mathrm{mm})$ between short implants and standard implants in augmented bone during loading (months) (random-effects model)

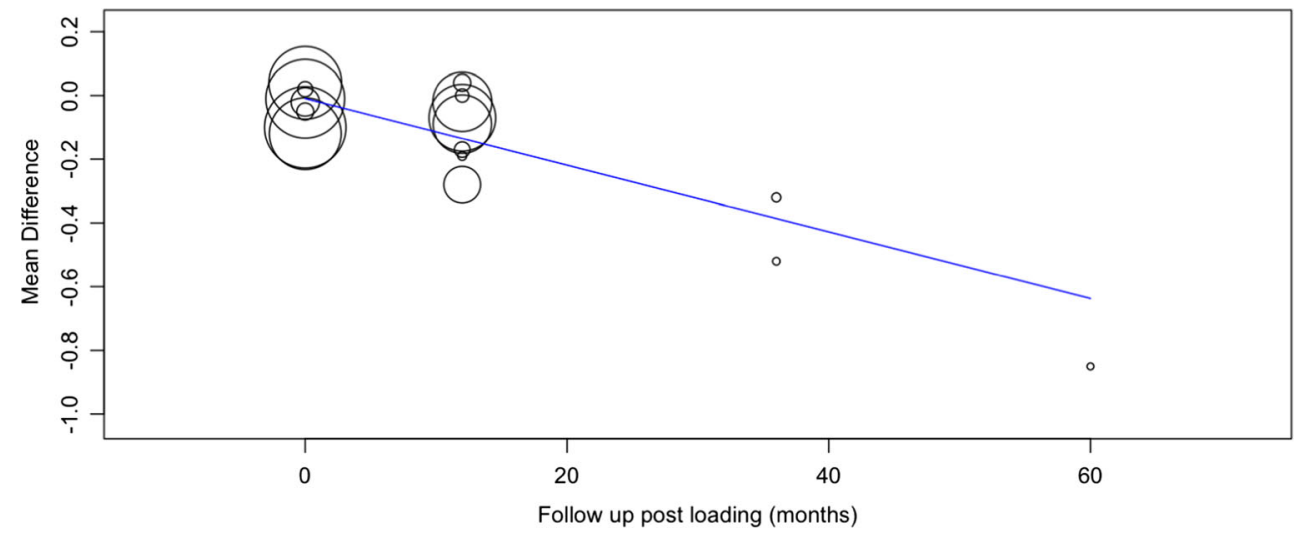




\section{Studies}

Pistilli, Felice, Piatelli, Esposito 2013

Pistilli, Felice, Cannizzaro, Esposito 2013

Felice, Cannizaro, Barausse, Esposito 2014

Esposito, Pistilli, Felice 2014

Gulj., Meijer 2014

Felice, Pistilli, Esposito 2015

Esposito, Barausse, Pistilli 2015

Overall $(95 \% \mathrm{Cl})(\mathrm{p}=0.82)$
Estimate (95\% C.I.) Ev/Trt Ev/Ctr

$0.500(0.046, \quad 5.385) \quad 1 / 68$

$0.227(0.011,4.662) \quad 0 / 80$

$1.017(0.310,3.333) 5 / 60$

$7.918(0.417,150.241) 3 / 60$

$0.318(0.014,7.383) 0 / 21$

$1.118(0.023,53.312) 0 / 16$

$1.069(0.021,53.288) \quad 0 / 86$

$0.902(0.381,2.137) \quad 9 / 391$

Fig. 6 Forest plot for prosthetic failure of the included studies. Ev/Trt represents the test (short implants) group, while $\mathrm{Ev} / \mathrm{Ctrl}$ represents the control (standard implants placed in grafted bone) group. The red line

These results are in agreement with previous studies for shorter implants [25]. Although the authors intended to compare fully edentulous with partially edentulous patients only one study was a RCT on fully edentulous patients [24] with a small sample size to be able to do statistical comparisons, however the results presented are in agreement with the studies with partially edentulous patients.

The number of implant failures was greater in the short term, either before prosthesis placement, or during the first months of loading. This could be due to the poor bone quality in which some of the implants were placed. Some authors suggest that lower quality of bone is associated with a higher failure rate [31] which is true in areas like the posterior maxilla or augmented bone $[32,33]$ so it would be expectable that the results of this SR presented higher failure rates when comparing to standard implants placed in native bone. However, two recently published meta-analysis reported annual failure rates between 2 and $3 \%[34,35]$ that are well within the values obtained in this systematic review for short implants and standard implants in augmented bone.

The presence of several confounding factors could not allow the authors to make a strong evidence based conclusions regarding the type of implant or regeneration material to be used, but the statistical analysis of the combined results suggests that the survival rate of both groups is similar with a relative risk of 1.34 between groups.

When comparing the results for the secondary outcomes, no significant differences were observed in mean differences of marginal bone at loading and prosthesis failure between both groups.

Some studies reported that the ratio crown-to-implant in short dental implants may lead to excessive occlusal and nonaxial loading which in turn may bring biological and technical complications [36-39], this relation has not been observed in this systematic review. However, these results should be view with caution since the information regarding the crown-to-implant ratio, the type of prosthesis and the use of splinting between crowns was scarce and could be considered a limitation of this study.

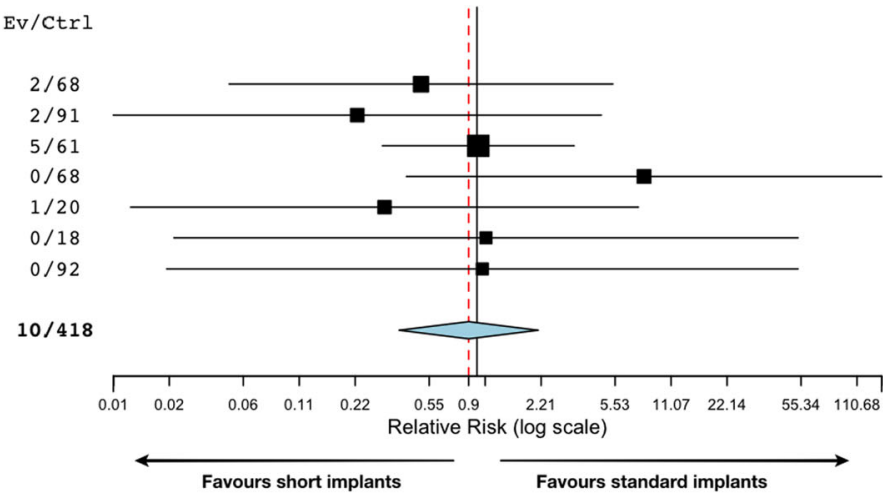

represents the average of all the results, and the vertical black line represents the no-effect line. Heterogeneity: $\mathrm{Tau}^{2}=0.00 ; \mathrm{Chi}^{2}=3.61, \mathrm{df}=6$ $(P=0.73) ; I^{2}=0 \%$

Sub-group analysis of mean differences in marginal bone changes in the different jaws, presented a statistical difference in the maxilla, marginally favouring the short implants, although with mean differences equating to $0.13-0.15 \mathrm{~mm}$ that are well bellow what can be considered of clinical significance.

When performing the meta-regression to evaluate the relationship between mean differences in MBL between both groups and follow-up period, it was possible to observe significant differences favouring the short implants group with smaller bone loss over time, with both groups not exceeding the criteria for MBL established for standard implants [40]. Hence, our findings point out that standard implants placed in regenerated bone have a higher bone loss over time when compared with short implants; however, this evidence is only corroborated by two available RCT. Several variables such as surgical technique [41, 42], implant design and connection [43], prosthetic considerations [28] or patient-centred variables [44] have to be considered aswell.

Despite the high quality of the included studies in this systematic review, there is a potential risk of bias due to the previous referred confounding factors and the small number of welldesigned studies with long follow up periods. Therefore, caution should be used when interpreting the results, but short implants can be used in atrophic bone areas with low vertical height with similar results to standard implants in augmented bone, at least in the short term (3-5 years).

\section{Conclusions}

Within the limitations of the present study, it has been found that short implants and standard-length implants in vertically augmented areas present comparable survival, prosthetic complication rates and marginal bone loss, being both techniques a correct option in order to replace one or more lost teeth. However, more randomised controlled trials with longer follow-up periods and bigger sample sizes have to be performed to confirm the present results. 
Author contribution All authors contributed equally to this systematic review.FundingThe work was supported by the Evidence Based Dentistry Center, Lisbon University and Implant Dentistry Department, European University of Madrid.

\section{Compliance with ethical standards}

Conflict of interest The authors declare that they have no conflict of interest.

Ethical approval This article does not contain any studies with human participants or animals performed by any of the authors.

Informed consent For this type of study, formal consent is not required.

\section{References}

1. Muneeb AKB, Jamil B (2013) Causes and pattern of partial edentulism/exodontia and its association with age and gender: semi rural population, Baqai Dental college, Karachi, Pakistan. Idjsr 1(3): 13-18

2. van der Velden U, Amaliya A, Loos BG, Timmerman MF, van der Weijden FA, Winkel EG, Abbas F (2015) Java project on periodontal diseases: causes of tooth loss in a cohort of untreated individuals. J Clin Periodontol 42(9):824-831. https://doi.org/10.1111/jcpe.12446

3. Baelum V, van Palenstein HW, Hugoson A, Yee R, Fejerskov O (2007) A global perspective on changes in the burden of caries and periodontitis: implications for dentistry. J Oral Rehabil 34(12):872-906; discussion 940. https://doi.org/10.1111/j.1365-2842.2007.01799.x

4. Madhankumar S, Mohamed K, Natarajan S, Kumar VA, Athiban I, Padmanabhan TV (2015) Prevalence of partial edentulousness among the patients reporting to the Department of Prosthodontics Sri Ramachandra University Chennai, India: an epidemiological study. J Pharm Bioallied Sci 7(Suppl 2):S643-S647. https://oi. org/10.4103/0975-7406.163580

5. Adell R, Lekholm U, Rockler B, Branemark PI (1981) A 15-year study of osseointegrated implants in the treatment of the edentulous jaw. Int J Oral Surg 10(6):387-416

6. Pennington JPS (2012) Improving quality of life using removable and fixed implant prostheses. Compend Contin Educ Dent 33(4): 268-270 272, 274-266

7. Karthik K, Sivakumar S, Thangaswamy V (2013) Evaluation of implant success: a review of past and present concepts. J Pharm Bioallied Sci 5(Suppl 1):S117-S119. https://doi.org/10.4103/09757406.113310

8. Santiago Junior JF, Pellizzer EP, Verri FR, de Carvalho PS (2013) Stress analysis in bone tissue around single implants with different diameters and veneering materials: a 3-D finite element study. Mater Sci Eng C Mater Biol Appl 33(8):4700-4714

9. Urban IA, Jovanovic SA, Lozada JL (2009) Vertical ridge augmentation using guided bone regeneration (GBR) in three clinical scenarios prior to implant placement: a retrospective study of $35 \mathrm{pa}-$ tients 12 to 72 months after loading. Int J Oral Maxillofac Implants 24(3):502-510

10. Monje A, Pikos MA, Chan HL, Suarez F, Gargallo-Albiol J, Hernandez-Alfaro F, Galindo-Moreno P, Wang HL (2014) On the feasibility of utilizing allogeneic bone blocks for atrophic maxillary augmentation. Biomed Res Int 2014:814578. https://doi.org/10. 1155/2014/814578

11. Pjetursson BE, Tan WC, Zwahlen M, Lang NP (2008) A systematic review of the success of sinus floor elevation and survival of implants inserted in combination with sinus floor elevation. J Clin Periodontol 35(8 Suppl):216-240. https://doi.org/10.1111/j.1600051X.2008.01272.x

12. Schwarz L, Schiebel V, Hof M, Ulm C, Watzek G, Pommer B (2015) Risk factors of membrane perforation and postoperative complications in sinus floor elevation surgery: review of 407 augmentation procedures. J Oral Maxillofac Surg 73(7):1275-1282. https://doi.org/10.1016/j.joms.2015.01.039

13. Jensen AT, Jensen SS, Worsaae N (2016) Complications related to bone augmentation procedures of localized defects in the alveolar ridge. A retrospective clinical study. Oral Maxillofac Surg 20(2): 115-122. https://doi.org/10.1007/s10006-016-0551-8

14. Raviv E, Turcotte A, Harel-Raviv M (2010) Short dental implants in reduced alveolar bone height. Quintessence Int 41(7):575-579

15. das Neves FD, Fones D, Bernardes SR, do Prado CJ, Neto AJ (2006) Short implants - an analysis of longitudinal studies. Int J Oral Maxillofac Implants 21(1):86-93

16. Neldam CA, Pinholt EM (2012) State of the art of short dental implants: a systematic review of the literature. Clin Implant Dent Relat Res 14(4):622-632. https://doi.org/10.1111/j.1708-8208. 2010.00303.x

17. Piero P, Lorenzo C, Daniele R, Rita G, Luca P, Giorgio P (2015) Survival of short dental implants $\leq 7 \mathrm{~mm}$ : A review. Int J Contemp Dent Med Rev 2015:Article ID: 011015. 10.15713/ins.ijcdmr.89

18. Egger MSG, Altman DG (2008) Chapter 2. Principles of and procedures for systematic reviews. BMJ Publishing Group, London. https://doi.org/10.1002/9780470693926.ch2

19. Pistilli R, Felice P, Piattelli M, Gessaroli M, Soardi E, Barausse C, Buti J, Corvino V (2013) Posterior atrophic jaws rehabilitated with prostheses supported by $5 \times 5 \mathrm{~mm}$ implants with a novel nanostructured calcium-incorporated titanium surface or by longer implants in augmented bone. One-year results from a randomised controlled trial. Eur J Oral Implantol 6(4):343-357

20. Pistilli R, Felice P, Cannizzaro G, Piatelli M, Corvino V, Barausse C, Buti J, Soardi E, Esposito M (2013) Posterior atrophic jaws rehabilitated with prostheses supported by $6 \mathrm{~mm}$ long $4 \mathrm{~mm}$ wide implants or by longer implants in augmented bone. One-year postloading results from a pilot randomised controlled trial. Eur J Oral Implantol 6(4):359-372

21. Felice P, Cannizzaro G, Barausse C, Pistilli R, Esposito M (2014 Winter) Short implants versus longer implants in vertically augmented posterior mandibles: a randomised controlled trial with 5year after loading follow-up. Eur J Oral Implantol. 7(4):359-369

22. Esposito M, Pistilli R, Barausse C, Felice P (2014 Winter) Threeyear results from a randomised controlled trial comparing prostheses supported by 5 -mm long implants or by longer implants in augmented bone in posterior atrophic edentulous jaws. Eur J Oral Implantol 7(4):383-395

23. Felice P, Pistilli R, Barausse C, Bruno V, Trullenque-Eriksson A, Esposito M (2015 Winter) Short implants as an alternative to crestal sinus lift: a 1-year multicentre randomised controlled trial. Eur J Oral Implantol. 8(4):375-384

24. Esposito M, Barausse C, Pistilli R, Sammartino G, Grandi G, Felice P (2015 Autumn) Short implants versus bone augmentation for placing longer implants in atrophic maxillae: one-year post-loading results of a pilot randomised controlled trial. Eur J Oral Implantol. $8(3): 257-268$

25. Thoma DS, Haas R, Tutak M, Garcia A, Schincaglia GP, Hämmerle CH (2015 Jan) Randomized controlled multicentre study comparing short dental implants $(6 \mathrm{~mm})$ versus longer dental implants (11-15 $\mathrm{mm}$ ) in combination with sinus floor elevation procedures. Part 1: demographics and patient-reported outcomes at 1 year of loading. J Clin Periodontol 42(1):72-80. https://doi.org/10.1111/jcpe.12323

26. Guljé FL, Raghoebar GM, Vissink A, Meijer HJA. (2014) Single crowns in the resorbed posterior maxilla supported by either 6-mm implants or by $11-\mathrm{mm}$ implants combined with sinus floor elevation 
surgery: A 1-year randomised controlled trial. Eur J Oral Implantol 7(3):247-55

27. Esposito M, Felice P, Worthington HV (2014) Interventions for replacing missing teeth: augmentation procedures of the maxillary sinus. Cochrane Database Syst Rev (5):CD008397. doi:https://doi. org/10.1002/14651858.CD008397.pub2

28. Koszuta P, Grafka A, Koszuta A, Lopucki M, Szymanska J (2015) Effects of selected factors on the osseointegration of dental implants. Prz Menopauzalny 14(3):184-187. https://doi.org/10.5114/ pm.2015.54343

29. Chambrone L, Preshaw PM, Ferreira JD, Rodrigues JA, Cassoni A, Shibli JA (2014) Effects of tobacco smoking on the survival rate of dental implants placed in areas of maxillary sinus floor augmentation: a systematic review. Clin Oral Implants Res 25(4):408-416. https://doi.org/10.1111/clr.12186

30. Bezerra Ferreira JD, Rodrigues JA, Piattelli A, Iezzi G, Gehrke SA, Shibli JA (2016) The effect of cigarette smoking on early osseointegration of dental implants: a prospective controlled study. Clin Oral Implants Res 27(9):1123-1128. https://doi.org/10.1111/ clr. 12705

31. Holahan CMWJ, Weaver A, Assad D, Koka S (2011) Relationship between systemic bone mineral density and local bone quality as effectors of dental implant survival. Clin Implant Dent Relat Res 13(1):29-33. https://doi.org/10.1111/j.1708-8208.2009.00206.x

32. Goiato MC, dos Santos DM, Santiago JF Jr, Moreno A, Pellizzer EP (2014) Longevity of dental implants in type IV bone: a systematic review. Int J Oral Maxillofac Surg 43(9):1108-1116. https:// doi.org/10.1016/j.ijom.2014.02.016

33. Nisand D (2000) Renouard F (2014) short implant in limited bone volume. Periodontol 66(1):72-96. https://doi.org/10.1111/prd. 12053

34. Engelhardt S, Papacosta P, Rathe F, Ozen J, Jansen JA, Junker R (2015) Annual failure rates and marginal bone-level changes of immediate compared to conventional loading of dental implants. A systematic review of the literature and meta-analysis. Clin Oral Implants Res 26(6):671-687. https://doi.org/10.1111/clr.12363

35. Chrcanovic BR, Albrektsson T, Wennerberg A (2015) Dental implants inserted in fresh extraction sockets versus healed sites: a systematic review and meta-analysis. J Dent 43(1):16-41. https:// doi.org/10.1016/j.jdent.2014.11.007

36. Isidor F (2006) Influence of forces on peri-implant bone. Clin Oral Implants Res 17(Suppl 2):8-18. https://doi.org/10.1111/j.16000501.2006.01360.x

37. Blanes RJ (2009) To what extent does the crown-implant ratio affect the survival and complications of implant-supported reconstructions? A systematic review. Clin Oral Implants Res 20(Suppl 4):67-72. https://doi.org/10.1111/j.1600-0501.2009.01762.x

38. Sotto-Maior BS, Senna PM, da Silva-Neto JP, de Arruda Nobilo MA, Del Bel Cury AA (2015) Influence of crown-to-implant ratio on stress around single short-wide implants: a photoelastic stress analysis. J Prosthodont 24(1):52-56. https://doi.org/10.1111/jopr. 12171

39. Ramos Verri F, Santiago Junior JF, de Faria Almeida DA, de Oliveira GB, de Souza Batista VE, Marques Honorio H, Noritomi PY, Pellizzer EP (2015) Biomechanical influence of crown-toimplant ratio on stress distribution over internal hexagon short implant: 3-D finite element analysis with statistical test. J Biomech 48(1):138-145. https://doi.org/10.1016/j.jbiomech.2014.10.021

40. Albrektsson TZG, Worthington P, Eriksson AR (1986) The longterm efficacy of currently used dental implants: a review and proposed criteria of success. Int J Oral Maxillofac Implants 1(1):11-25

41. Qian J, Wennerberg A, Albrektsson T (2012) Reasons for marginal bone loss around oral implants. Clin Implant Dent Relat Res 14(6): 792-807. https://doi.org/10.1111/cid.12014

42. Sanz M, Vignoletti F (2015) Key aspects on the use of bone substitutes for bone regeneration of edentulous ridges. Dent Mater 31(6):640-647. https://doi.org/10.1016/j.dental.2015.03.005

43. Monje A, Suarez F, Galindo-Moreno P, Garcia-Nogales A, Fu JH, Wang HL (2014) A systematic review on marginal bone loss around short dental implants $(<10 \mathrm{~mm})$ for implant-supported fixed prostheses. Clin Oral Implants Res 25(10):1119-1124. https://doi. org $/ 10.1111 /$ clr. 12236

44. Galindo-Moreno P, Fauri M, Avila-Ortiz G, Fernandez-Barbero JE, Cabrera-Leon A, Sanchez-Fernandez E (2005) Influence of alcohol and tobacco habits on peri-implant marginal bone loss: a prospective study. Clin Oral Implants Res 16(5):579-586. https://doi.org/ 10.1111/j.1600-0501.2005.01148.x 